\title{
Comparison between hypoxic markers pimonidazole and glucose transporter 1 (Glut-1) in murine fibrosarcoma tumours after electrochemotherapy
}

\author{
Andrej Cör ${ }^{1}$, Maja Cemazar ${ }^{1,2}$, Nadja Plazar ${ }^{1}$, Gregor Sersa $^{2}$ \\ ${ }^{1}$ College of Health Care Izola, University of Primorska, Izola, Slovenia \\ ${ }^{2}$ Institute of Oncology Ljubljana, Zaloska 2, Ljubljana, Slovenia
}

\begin{abstract}
Background. Tumour hypoxia occurs as a result of an inadequate supply of blood-borne oxygen due to the disorganized and chaotic vascular network that develops in tumours. Because tumour hypoxia has been associated with a more aggressive phenotype and lower cure rate, there is a recognized need for a method of measuring tumour hypoxia that is suitable for widespread clinical use. The aim of the current study was to compare the expression of Glut-1 with the binding of the bioreductive hypoxia marker pimonidazole and to elucidate the characteristics and pitfalls when they are used as hypoxic markers.

Materials and methods. In the study, SA-1 solid subcutaneous tumours in A/J mice were treated by bleomycin given i.v. $(1 \mathrm{mg} / \mathrm{kg})$, or the application of electric pulses (8 pulses, $1400 \mathrm{~V}, 100 \mathrm{\mu s}, 1 \mathrm{~Hz}$ ), or a combination of the two - electrochemotherapy. Pimonidazole was injected 16 hours before tumour excision. Tumours were excised at different time points (0.5, 1, 2, 8, 14 and 24 hours) after therapy. Immunohistochemistry for Glut-1 and pimonidazole adduct was carried out on two consecutive tumour sections and the percentages of positive staining areas were determined.
\end{abstract}

Results. Glut-1 staining was membranous and typically expressed peri-necrotically, whereas pimonidazole staining, although showing substantial co-localisation with Glut-1, was cytoplasmatic. More than 65\% of the stained areas showed a high degree of colocalization when the two markers were compared. Our results show that Glut-1 expression significantly correlates with the level of pimonidazole binding $(r=0.41 ; p=$ 0.028).

Conclusions. Our study confirms that HIF-1 regulated genes, such as Glut-1, have potential for future use as predictors of a decreased sensitivity of tumours to radio- and chemotherapy mediated by hypoxia.

Key words: hypoxia; pimonidazole; Glut-1; electrochemotherapy

\section{Introduction}

Received 1 July 2009

Accepted 19 August 2009

Correspondence to: Prof. Andrej Cör, MD, PhD, College of Health Care Izola, University of Primorska, Polje 42, 6310 Izola, Slovenia. Phone: +38656626471; E-mail: andrej.coer@vszi.upr.si
Measurements of oxygen partial pressure in tumours performed with microelectrodes have yielded novel information concerning the contribution of the tumour 
oxygenation status to the course of malignant growth and have shown that the presence of hypoxic areas to be a universal characteristic of solid malignant tumours. ${ }^{1}$ Tumour hypoxia, mostly resulting from poor perfusion and anaemia, is one of the key factors for induction and development of tumour cell clones with an aggressive and treatment resistant phenotype, which leads to rapid tumour progression and poor prognosis. $^{2,3}$ Tumour hypoxia has an impact on such fundamental aspects of malignancy as a) cell survival and proliferation, b) angiogenesis, c) cancer cell invasiveness, d) metastasis, e) resistance to apoptosis, and $\mathrm{f}$ ) genetic instability. ${ }^{4}$ The expression of more than 70 genes is altered under hypoxic conditions, as a result of the change in stability of a critical transcription factor called hypoxia inducible factor-1 (HIF-1), which allows a tumour to survive the harsh tumour microenvironment.

Direct assessment with oxygen electrodes is often referred to as a gold standard, but is still considered to be an experimental tool. Since $\mathrm{O}_{2}$ microelectrode measurements are invasive and applicable only to tumour entities accessible to needle electrodes, there is great interest in substitute methods for assessing of the oxygenation status. Bioreductive markers, such as pimonidazole, provide an alternative approach for assessing of the level and extent of tumour hypoxia. Pimonidazole, administered approximately $16 \mathrm{~h}$ prior to tumour excision, is reductively activated in an oxygen-dependent manner and is covalently bound to thiol-containing proteins in hypoxic cells, forming intracellular adducts that can be detected immunohistochemically. ${ }^{5,6}$ A clinical comparative study has recently demonstrated a correlation between microelectrode measurements of $\mathrm{pO}_{2}$ and the extent of pimonidazole adduct formation in carcinoma of the cervix. ${ }^{7}$ However, studies on archived material are not possi- ble because the drug must be administered prospectively.

There is an increasing need for endogenous markers to assess the presence of hypoxia. Endogenous markers would have additional advantages, since they do not require the application of a foreign substance and would allow studies of oxygenation status in archival paraffin material. ${ }^{8}$ Glucose transporter- 1 (Glut-1) is one of the proteins upregulated in a hypoxic condition. In a tumour microenvironment, hypoxia results in an increased transcription of the Glut-1 gene, mediated by HIF-1. Tumours show increased uptake of glucose compared to normal tissue and Glut- 1 is responsible for the passive transport of glucose across the cell membrane. ${ }^{9}$ Glut-1 over-expression has been associated with enhanced tumour aggressiveness and an unfavourable clinical outcome in various tumour types. ${ }^{4}$ It has been suggested that Glut-1 might represent an intrinsic marker of hypoxia. ${ }^{10}$

The goal of our study was to compare the expression of Glut-1 with the binding of the bioreductive hypoxia marker pimonidazole and to elucidate the characteristics and pitfalls when each of them is used as a hypoxic marker. For this purpose, solid subcutaneous murine SA-1 sarcomas were treated with hypoxia-inducible therapy (electrochemotherapy) and excised at different posttreatment times for the determination of selected markers of hypoxia.

\section{Materials and methods}

Murine fibrosarcoma SA-1 cells and A/J mice were used for this study. Solid subcutaneous tumours, located dorsolaterally in mice, were initiated by an injection of $5 \times 10^{5}$ SA- 1 cells in $0.1 \mathrm{ml} 0.9 \% \mathrm{NaCl}$ solution. Six to 8 days after implantation, when the tumours had reached approximately $40 \mathrm{~mm}^{3}$ in volume, the mice were randomly divided 
into experimental groups. In the first group, bleomycin (Heinrich Mack Nachf.,Illertisen, Germany) at a dose of $1 \mathrm{mg} / \mathrm{kg}$ was injected intravenously. In the second group, eight square electric pulses of $100 \mu \mathrm{s}$ (at a voltage to distance ratio of $1400 \mathrm{~V} / \mathrm{cm}$ ) were delivered by two flat electrodes $8 \mathrm{~mm}$ apart. In the electrochemotherapy group, the mice were treated with electric pulses 3 minutes after bleomycin injection. Tumours without treatment were used as controls. The tumours were excised at different time points $(0.5,1,2,8,14$ and $24 \mathrm{~h})$ after treatment with bleomycin, the application of electric pulses or electrochemotherapy. Pimonidazole $\mathrm{HCl}$ (Hypoxyprobe-1, Natural Pharmacia International Inc.) was administered intraperitoneally to mice at a single dose of $100 \mathrm{mg} / \mathrm{kg} 16 \mathrm{~h}$ before sacrifice and tumour excision. Treatment protocol was approved by the Ministry of Agriculture, Forestry and Food of the Republic Slovenia No. 3440-12/2009/6.

Tumour tissue specimens were formalinfixed and paraffin-embedded. Two consecutive $5 \mu \mathrm{m}$ thick sections were cut from each paraffin block for immunohistochemical analysis. Immunoperoxidase with diaminobenzidine tetrahydrochloride (DAB) substrate was performed to detect hypoxic regions indicated by the presence of pimonidazole or Glut-1. Sections were dewaxed and hydrated in graded alcohols, rinsed in distilled water and PBS and treated with $3 \%$ hydrogen peroxide in methanol for 10 min to eliminate endogenous peroxydase activity and thereby prevent non-specific reactions with $\mathrm{DAB}$ substrate. Sections for Glut-1 detection were pre-treated in a pressure cooker in citric buffer ( $\mathrm{pH}$ 6.0) and allowed to cool at room temperature for 15 minutes. Fifty microlitres of mouse monoclonal antibody raised against intracellular pimonidazole adduct (Natural Pharmacia International Inc) at a dilution 1/100 for $2 \mathrm{~h}$ or anti-Glut-1 (Alpha Diagnostic
International) at a dilution of $1 / 100$ for $1 \mathrm{~h}$ was applied. An EnVision kit (Dako, UK) was used for primary antibody binding detection. Immunoreactivity was visualized with DAB, and hematoxylin was used as a counterstain. Erythrocytes in each section served as positive controls for Glut-1. As negative controls, adjacent sections were incubated in parallel with non-immune serum instead of the primary antibodies.

An Eclipse-80i (Nikon, Japan) light microscope with an attached CCD camera was used for acquisition of images of stained tumour sections. A semi-quantitative scoring system was applied to the Glut-1 and pimonidazole adduct stained sections, and a score of 0-4 was assigned for each microscopic field, representative of the approximate area of immunostaining: 0 negative, $1+<5 \%, 2+5-15 \%, 3+15-40 \%$ and $4+>40 \%$. Areas of necrosis, stroma, normal skin over the tumour and distinct edge effects were ignored. The overall scores used in our study summarising pimonidazole binding or Glut-1 expression across the tumour was derived from the average score for all fields. Two-tailed, Spearman's rank correlations were used to assess the relationship between the hypoxia markers used in our study. A $P$ value less than or equal to 0.05 was considered statistically significant.

\section{Results}

Electrochemotherapy with bleomycin has been very effective in the treatment of subcutaneous SA-1 tumours, resulting in substantial tumour growth delay and even a high percentage of tumour cures, compared to untreated tumours and tumours treated with bleomycin or application of electric pulses only, as also demonstrated in our previous study. ${ }^{11}$

In the present study we compared two hypoxia markers and two consecutive sec- 


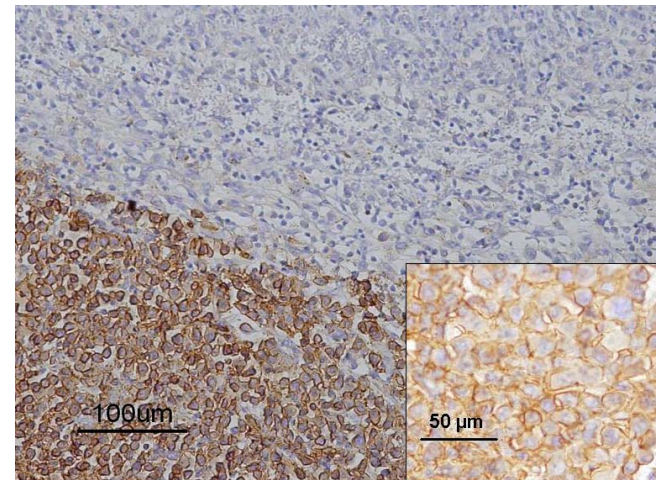

Figure 1. Immunohistochemical detection of Glut-1 in murine fibrosarcoma tumours after electrochemotherapy. Differences between Glut-1 positive and Glut-1 negative tumour areas are seen on large picture. Glut-1 expression is detected as membranous staining (small square).

tions from each tumour were therefore analysed. Glut-1 expression and pimonidazole binding were visualised by immunohistochemistry. The staining intensities for both markers generally increased in the vicinity of the necrotic area. Normal skin over the tumour failed to stain for both markers. However, strong immunoractivity exclusively to Glut-1 was found in red blood cells. A positive reaction for Glut-1 was seen in both cytoplasm and the tumour cell membrane. As the staining score increased, the cell membrane became more stained (Figure 1). Pimonidazole staining was found in the cytoplasm of tumour cells (Figure 2). Much of the staining was concentrated around necrotic tissue, and regions that were positive for pimonidazole were generally positive for Glut-1, although the Glut-1 expression areas were narrower in extent than the pimonidazole binding areas.

In the control and bleomycin groups the area of tumour hypoxia was approximately $10 \%$ at all time-points examined. After application of electric pulses alone, as well as after electrochemotherapy, the extent of tumour hypoxia detected by Glut-1 or pimonidazole reached its peak after $2 \mathrm{~h}$ (Figure 3)

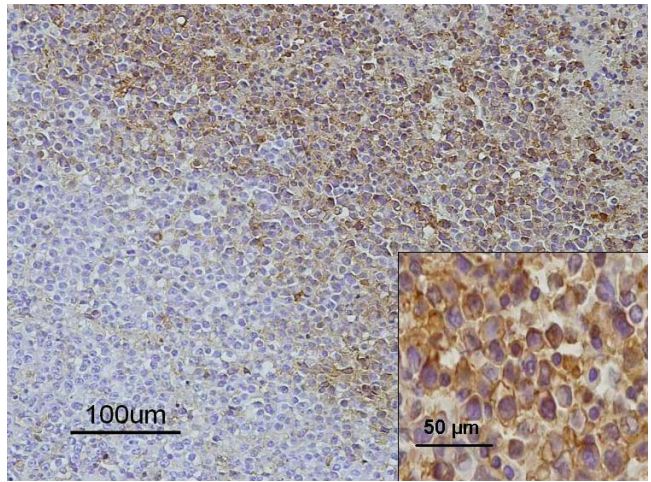

Figure 2. Immunohistochemical detection of pimonidazole bindig in murine fibrosarcoma tumours after electrochemotherapy. Differences between pimonidazole positive and pimonidazole negative tumour areas are seen on large picture. Pimonidazole bindig is detected as cytoplasmatic staining (small square).

and lasted up to $8 \mathrm{~h}$ after the treatment. After the application of electric pulses, the recovery to pre-treatment level occurred at $14 \mathrm{~h}$, whereas after electrochemotherapy the hypoxic area started to decrease after $14 \mathrm{~h}$. However, recovery to pre-treatment level did not occur for at least $24 \mathrm{~h}$ (Figure 4).

The mean and range of the marker positive fraction varied between the two markers. However, there were significant correlations $(r=0.41)$ between Glut-1 and pimonidazole scores $(p=0.028)$. The degree of co-localisation between stained regions is also critical in their evaluation as hypoxia markers. More than $65 \%$ of the stained areas showed a high degree of co-localization when the two markers were compared.

\section{Discussion}

We used two hypoxic markers, pimonidazole and Glut-1, to detect hypoxic areas of SA-1 tumours treated with hypoxia-inducible therapy (electrochemotherapy). We found that there is a correlation between exogenous hypoxic marker pimonidazole binding and endogenous hypoxia marker 

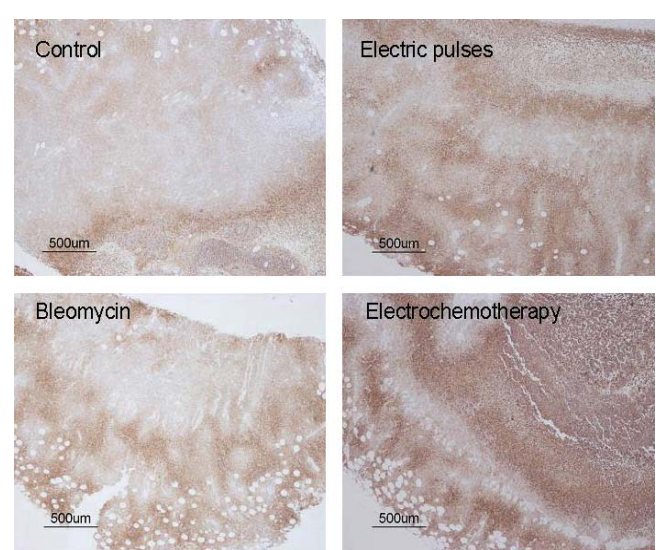

Figure 3. Glut-1 expression in representative sections of mourine fibrosarcoma tumours two hours after therapy with bleomycin, application of electric pulses or electrochemotherapy.

Glut-1 expression, so immunohistochemical staining of endogenous hypoxic marker can be used to track changes in tumour hypoxia. Our results resemble those of Russell et al., who have previously shown that labelling hypoxic areas with the endogenous hypoxic marker carbonic anhydrase (CA) IX can be used to detect changes in tumour xenograft oxygenation. ${ }^{5}$ Glut-1 and CAIX are two downstream genes regulated by hypoxia-inducible factor (HIF)- $1 \alpha$ and both are considered to be endogenous hypoxia markers.

There is an intense effort to develop techniques that are able to visualize and quantify tumour hypoxia, because hypoxia can cause resistance to radiotherapies (hypoxic cells are 2-3 fold more radio-resistant than aerated cells) ${ }^{12}$ and promote malignant progression. Hypoxic cells are also less accessible to nutrients and drugs, more likely to be non-cyclic, and therefore resistant to many forms of chemotherapy. ${ }^{13,14}$ Currently, the two methods most widely used for direct measurement of tumour oxygenation are an Eppendorf polarographic oxygen electrode and the luminescence-based optical sensor OxyLite. ${ }^{15}$ The drawback of those methods are their invasiveness and that they can

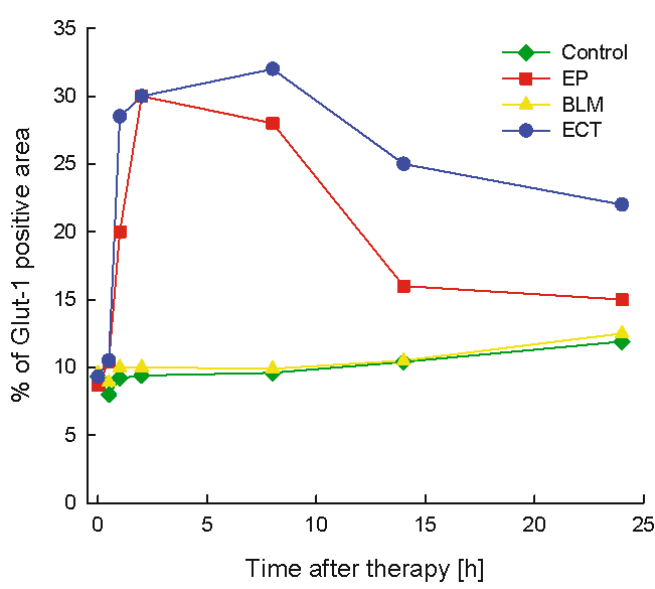

Figure 4. Time course of Glut-1 positive area in control tumours and after treatments with bleomycin (BLM), electric pulses (EP) and electrochemotherapy (ECT).

not distinguish a hypoxic region in viable tumour tissue from a low oxygen level in necrotic tumour areas.

An alternative method is immunohistochemical assessment of pimonidazole binding, injected prior to the biopsy being taken. ${ }^{16}$ The advantage of the immunohistochemical marker approach is that the same or contiguous formalin-fixed tissue sections may be examined for relationships between hypoxia and other physiological parameters for which immunohistochemical assays exist. In addition, histological sections permit the study of the geographical distribution of hypoxia, and micro-environmental factors such as blood vessels, areas of cell proliferation and angiogenesis. ${ }^{17}$ Pimonidazole has been used for hypoxia assessment with various xenograft tumour treatment protocols ${ }^{12,18}$, but pimonidazole must be administered 16 to $24 \mathrm{~h}$ before tumour biopsy and it is likely to reflect the presence of more long term or chronic hypoxia or, put in another way, pimonidazole may not indicate the level of acute hypoxia. ${ }^{19}$

The discovery that tumours metabolise sugars at an increased rate to normal tissue was made over 70 years ago. Cancer cell energy metabolism has received inter- 
est recently, with some common oncogenic mutations having been directly linked to the glycolytic phenotype. ${ }^{18}$ Glut- 1 is one of the glucose transporters located in the cell membrane. It is known that increased expression of Glut-1 is not only found in a wide variety of tumour types, but invariably indicates a poor prognosis. ${ }^{20,21}$ It has also been suggested that Glut-1 might represent an intrinsic marker of hypoxia. ${ }^{14,17}$ There is a fundamental drawback, in that Glut-1 expression does not exclusively correspond to the tumour oxygenation status. Rather, the expression in tumour cells might reflect the activation of other oncogenic pathways, independent of hypoxia. In fact, two recent reports comparing oxygen tension in cervical cancers to HIF-1 $\alpha$ and Glut-1 expression provided strong evidence for a regulation of these pathways independently of hypoxia.9,22 However, Kunkel et al. recently confirmed the value of Glut-1 expression as a predictive marker for radio-resistant squamous cell carcinoma of the oral cavity. In addition to providing prognostic information, data suggest that modulation of radiation resistance by inhibition of glucose transport in the tumour may be a novel strategy for improving the effectiveness of radiotherapy in tumours. ${ }^{23}$ The advantage of using intrinsic markers of hypoxia, such as Glut-1, is that the approach is simple and quick, and could potentially be applied to a wide variety of solid tumour types.

Our results indicate that pimonidazole and Glut-1 show a similar ability for detecting hypoxic cells. The mean tumour area of marker positivity varied between the two markers; however, there was a correlation between them. A small number of studies have compared endogenous marker expression with either oxygen microelectrode measurements or exogenous hypoxia marker binding. There are some reports of a lack of correlation between oxygen electrode measurements and Glut-1 expression ${ }^{24}$ or pimonidazole binding. ${ }^{25}$ In contrast, two recent studies have shown that levels of Glut-1 expression in carcinoma of the cervix correlated with the level of tumour hypoxia, measured using either polarographic needle electrodes ${ }^{21}$ or pimonidazole staining. ${ }^{14} \mathrm{~A}$ strong correlation was also observed between pimonidazole and Glut-1 in human bladder cancer and Glut-1 was an independent prognostic factor for overall survival of these patients. ${ }^{22}$ The relationship between marker expression and oxygen electrode measurement is a complex one, since these methods do not sample the same tumour micro-environment or provide directly comparable measures of hypoxia. A general conclusion has been that endogenous markers offer promise for the routine measurement of tumour hypoxia but they may not provide the same information as $\mathrm{O}_{2}$ measurements using microelectrodes or binding of chemical hypoxia markers. $^{26}$

The induction of tumour hypoxia has often been intuitively linked to an inability of the tumour vascular network to provide a nutritive blood supply to the rapidly proliferating tissue. Highly angiogenic tumours should thus be well oxygenated. However, several studies have indicated that, paradoxically, tumour hypoxia correlates with high vascular density in vivo. ${ }^{27,28}$ It is likely that several factors contribute to tumour hypoxia. During the rapid growth of a tumour, an aberrant microvasculature develops. The abnormal structure and function of the tumour microvasculature will reduce perfusion, increase the amount of fluid leaking into the extravascular space of the tumour, and thus increase the viscous resistance to flow. There is currently great interest in the relationship between tumour hypoxia and tumour vasculature because tumour blood vessels are an attractive target for tumour therapy. 
In our study, we analysed tumours treated with electric pulses and/or bleomycin. After electrochemotherapy the hypoxic tumour area (determined with pimonidazole or Glut-1) increased to 35\% within $1 \mathrm{~h}$ after treatment, reaching a peak $2 \mathrm{~h}$ after treatment. The antitumour efficiency of electrochemotherapy is not only due to increased cytotoxicity but also due to an anti-vascular effect, which results in reduced tumour blood flow and increased tumour cell hypoxia. ${ }^{29}$ It has been proposed that electrochemotherapy, in addition to the direct cytotoxic effect, also has a vascular disrupting action. $^{11}$

\section{Conclusions}

The correlations between the endogenous hypoxia marker Glut-1 and pimonidazole binding established in this study confirm that intrinsic markers of hypoxia, such as Glut-1, are a reliable means of evaluating tumour hypoxia, which will continue to be useful in future investigations involving archival material from a range of sources. However, application of these markers requires careful validation against established methods, especially when many other factors could complicate the use of hypoxia responsive gene expression as an indication of tumour hypoxia.

\section{Acknowledgement}

The authors acknowledge the financial support of the state budget by Slovenian Research Agency (Program No. P3-0003). All the authors declare that they have no conflict of interest.

\section{References}

1. Kranjc S, Cemazar M, Grosel A, Sentjurc M, Sersa G. Radisensitising effect of electrochemotherapy with bleomycin in LPB sarcoma cells in tumors in mice. BMC Cancer 2005; 5: 115.

2. Teicher BA. Acute and chronic in vivo therapeutic resistence. Biochem Pharmacol 2009; 77: 1665-73.

3. Rademakers SE, Span PN, Kaanders J, Swep F, van der Kogel AJ, Bussink J. Molecular aspects of tumour hypoxia. Mol Oncol 2008; 2: 41-53.

4. Mayer A, Höckel M, Wree A, Vaupel P. Lack of correlation between expression of HIF- $1 \alpha$ protein and oxygenation status in identical tissue areas of squamous cell carcinomas of the uterine cervix. Cancer Res 2004; 64: 5876-81.

5. Russell J, Carlin S, Burke SA, Wen B, Yang $\mathrm{KM}$, Ling CC. Immunohistochemical detection of changes in tumor hypoxia. Int J Radiat Oncol Biol Phys 2009; 73: 1177-86.

6. Raleigh JA, Chou SC, Bono EL, Thral DE, Varia MA. Semiquantitative immunohistochemical analysis for hypoxia in human tumours. Int $J$ Radiat Oncol Biol Phys 2001; 49: 569-74.

7. Nordsmark M, Loncaster J, Chou SC, Hacsteen H, Lidegaard JC, Davidson SE, et al. Invasive oxygen measurements and pimonidazole labelling in human cervix carcinoma. Int J Radiat Oncol Biol Phys 2001; 49: 581-6.

8. Sakata K, Someya M, Nagakura H, Nakata K, Oouchi A, Hareyama M, et al. A clinical study of hypoxia using endogenous hypoxic markers and polarographic oxygen electrodes. Strahlenther Oncol 2006; 182: 511-7.

9. Chen C, Pore N, Behrooz A, Ismail-Beigi F, Maity A. Regulation of glut-1 mRNA by hypoxia inducible factor-1. J Biol Chem 2001; 276: 9519-25.

10. Cooper R, Sarioglu S, Sökmen S, Füzün M, Küpelioglu A, Valentine A, et al. Glucose transporter-1 (GLUT-1): a potential marker of prognosis in rectal carcinoma. Br J Cancer 2003; 89: 870-6.

11. Sersa G, Jarm T, Kotnik T, Coer A, Podkrajsek $M$, Sentjurc $M$, et al. Vascular disrupting action of electroporation and electrochemotherapy with bleomycin in murine sarcoma. Br J Cancer 2008; 98: 388-98. 
12. Sun X, Li XF, Russell J, Xing L, Urano M, Li GC, et al. Changes in tumor hypoxia induced by mild temperature hyperthermia as assessed by dualtracer immunohistochemistry. Radiother Oncol 2008; 88: 269-76.

13. Airley RE, Philips RM, Evans AE, Double J, Burger $\mathrm{AM}$, Feibig $\mathrm{HH}$, et al. Hypoxia-regulated glucose transporter Glut-1 may influence chemosensitivity to some alkylating agents: results of EORTC (first translational award) study of the relevance of tumour-hypoxia to the outcome of chemotherapy in human tumour derived xenografts. Int J Oncol 2005; 26: 1477-84.

14. Airley RE, Loncaster J, Raleigh JA, Harris AL, Davidson SE, Hunter RD, et al. Glut-1 and CAIX as intrinsic markers of hypoxial in carcinoma of the cervix: Relationship to pimonidazole binding. Int J Cancer 2003; 104: 85-91.

15. Jarm T, Sersa G, Miklavcic D. Oxygenation and blood flow in tumours treated with hydralazine: Evaluation with a novel luminescence-based fiberopric sensor. Technol Health 2002; 10: 363-80.

16. Kennedy AS, Raleigh JA, Perez GM, Calkins DP, Thrall DE, Novotny DB, et al. Proliferation and hypoxia in human squamous cell carcinoma of the cervix: first report of combined immunohistochemical assays. Int J Radiat Oncol Biol Phys 1997; 37: 897-905.

17. Olive PL, Durand RE, Raleigh JA, Luo C, AquinoParsons C. Comparison between the comet assay and pimonidazole binding for measuring tumour hypoxia. Br J Cancer 2000; 83: 1525-31.

18. He F, Deng X, Wen B, Liu Y, Sun X, Xing L, et al. Nonivasive molecular imaging of hypoxia in human xenografrts: comparing hypoxia-induced gene expression with endogenous and exogenous hypoxia markers. Cancer Res 2008; 68: 8597-606.

19. Gulliksnud K, Vestvik IK, Galapathi K, Rofstad EK. Detection of different hypoxic cell subpopulations in human melanoma xenografts by pimonidazole immunohistochemistry. Radiol Res 2008; 170: $638-50$.

20. Rajaganeshan R, Prased R, Guillou PJ, Poston G, Scott N, Jayne DG. The role of hypoxia in recurrence following resection of Dukes'B colorectal cancer. Int J Colorectal Dis 2008; 23: 1049-55.

21. Yasuda M, Miyazawa M, Fujita M, Kajiwara $H$, Iida T, Hirasawa T, et al. Expression of hypoxia inducible factor 1-alfa (HAF-1alfa) and glucose transporter 1 (GLUT-1) in ovarian adenocarcinoma difference in hypoxic status depending on histological character. Oncol Rep 2008; 19: 111-6.
22. Hoskin PJ, Sibtain A, Daley FM, Wilson GD. GLUT 1 and CAIX as intrinsic markers of hypoxia in bladder cancer: relationship with vascularity and proliferation as predictors of outcome of ARCON. Br J Cancer 2003; 89: 1290-7.

23. Kunkel M, Moergel M, Stockinger M, Joeng JH, Fritz G, Lehr HA, et al. Overexpression of Glut-1 is associated with resistence to radiotherapy and adverse prognosis in squamous cell carcinoma of the oral cavity. Oral Oncol 2007; 43: 796-803.

24. Mayer A, Hockel M, Wree A, Vaupel P. Microregional expression of glucose transporter 1 and oxygenation status lack of correlation in locally advanced cervical cancer. Clin Cancer Res 2005; 11: 2768-73.

25. Hedley D, Pintilie M, Woo J, Morrison A, Birle D, Fyles A, et al. Carbonic anhidrase IXexpression, hypoxia, and progression in patients with uterin cervical carcinomas. Clin Cancer Res 2003; 9: 566674.

26. Jankovic B, Aquino-Persons C, Raleigh JA. Comparison between pimonidazole binding oxygen electrode measurements, and expression of endogenous hypoxia markers in cancer of the uterine cervix. Cytometry 2006; 70B: 45-55.

27. Koukourakis MI, Giatromanolaki A, Sivridis E, Pastorek J, Karapantzos I, Gatter KC, et al. Hypoxia-actovated tumor pathways of angiogenesis and regulation of $\mathrm{pH}$ independent of anemia in head-and-neck cancer. Int J Radiat Oncol Biol Phys 2004; 59: 67-71.

28. Kostourou V, Troy H, Murray JF, Cullis ER, Whitley GS, Griffiths JR, et al. Overexpression of dimethylarginine dimethylaminohydrolase enhances tumour hypoxia: An insight into the relationship of hypoxia and angiogenesis in vivo. Neoplasia 2004; 6: 401-11.

29. Teissie J, Escoffre JM, Rols MP, Golzio M. Time dependence of electric field effects on cell membranes. A review for a critical selection of pulse duration for therapeutical applications. Radiol Oncol 2008; 42: 196-206.

30. Zupancic A, Corovic S, Miklavcic D. Optimization of electrode position and electric pulse amplitude in electrochemotherapy. Radiol Oncol 2008; 42: 93101.

31. Sersa G, Krzic M, Sentjurc M, Ivanusa T, Beravs K, Kotnik V, et al. Reduced blood flow and oxygenation in SA-1 tumours after electrochemotherapy with cisplatin. Br J Cancer 2002; 87: 1047-54. 